Voix et Images

volxetimages

\title{
Un témoin dérangeant. À propos de Pierre Vadeboncoeur
}

\section{Stéphane Inkel}

Volume 38, numéro 2 (113), hiver 2013

Louis Dantin

URI : https://id.erudit.org/iderudit/1015169ar

DOI : https://doi.org/10.7202/1015169ar

Aller au sommaire du numéro

Éditeur(s)

Université du Québec à Montréal

ISSN

0318-9201 (imprimé)

1705-933X (numérique)

Découvrir la revue

Citer ce compte rendu

Inkel, S. (2013). Compte rendu de [Un témoin dérangeant.

À propos de Pierre Vadeboncoeur]. Voix et Images, 38(2), 125-131.

https://doi.org/10.7202/1015169ar d'utilisation que vous pouvez consulter en ligne.

https://apropos.erudit.org/fr/usagers/politique-dutilisation/ 


\author{
E S S A I S / É T U D E S \\ Un témoin dérangeant \\ $\bar{A}$ propos de Pierre Vadeboncœur \\ $+++$ \\ STÉPHANE INKEL \\ Université Queen's
}

Prenant prétexte d'une lecture de ses Essais sur la croyance et l'incroyance par Étienne Beaulieu dans les cahiers Contre-jour ${ }^{1}$, Pierre Vadeboncœur y va, dans La clef de voûte $^{2}$, d'une réflexion sur le genre de l'essai, qu'il pratique depuis plus d'un demisiècle, genre littéraire particulier en ceci que son matériau premier est fait d'idées, ce qui l'inscrit dans le registre de l'opinable. Rappelant l'essai à sa nature d'écrits et au style qu'il présuppose, Vadeboncœur avance le paradoxe suivant pour définir son travail: «Même si je discute, je ne discute pas...» $(C V, 152)$ Je laisse aux spécialistes du genre le soin de commenter, et au besoin de contester, les implications d'une telle définition de l'essai. Par contre, les conséquences d'une telle définition pour la critique devraient ici nous intéresser, elle qui se voit confinée par Vadeboncœur à une mesure d'accompagnement de l'œuvre d'art, à sa mise en valeur et à son accueil plutôt qu'à sa réfutation, voire même à sa discussion (CV, 153-154). Cette chronique ne porte pas sur l'œuvre de Vadeboncœur, mais sur deux monographies qui viennent de lui être consacrées: Un moderne à rebours ${ }^{3}$ de Jonathan Livernois et $D^{\prime}$ un royaume à l'autre $^{4}$ de Réjean Beaudoin. Il s'agit par conséquent d'une critique au deuxième degré. À ce titre, et en cherchant à faire écho à la fonction qui lui est prêtée par Vadeboncœur, j'aimerais, pour débuter, non pas définir la critique, mais poser une première pierre en ce sens qui puisse nous permettre d'appréhender ces deux ouvrages et d'en mesurer la finalité. En ce qui concerne les monographies, il s'agirait de soutenir que la critique ne consiste pas tant à accueillir les œuvres dans son propre discours qu'à tisser leur parole respective afin de les inscrire (ou de les réinscrire) sur la scène de la délibération de l'espace public, et de faire en sorte que celui-ci s'en trouve visé. De ce point de vue, les deux ouvrages dont il sera ici question représentent les pôles

$$
+++
$$

1 Étienne Beaulieu, «La communauté ignorée», Contre-jour. Cahiers littéraires, n 9, printemps 2006, p. 181-186.

2 Pierre Vadeboncœur, La clef de voûte, Montréal, Bellarmin, 2008, 168 p. Désormais, les références à cet ouvrage seront indiquées par le sigle $C V$ suivi du folio, et placées entre parenthèses dans le texte. 3 Jonathan Livernois, Un moderne à rebours. Biographie intellectuelle et artistique de Pierre Vadeboncœur, Québec, Presses de l'Université Laval, coll. «Cultures québécoises», 2012, 355 p. 4 Réjean Beaudoin, D'un royaume à l'autre. Essai sur Pierre Vadeboncœur, Montréal, Leméac, 2012, 229 p. 
opposés de cette tension discursive, l'un toujours enclin à retrouver sous les idées de Vadeboncœur, y compris les dernières, les échos des discours ayant circulé dans la société contemporaine de ses années de formation, alors que l'autre, celui de Beaudoin, s'approprie la parole, inactuelle, de l'essayiste pour mieux la donner à entendre en fonction des enjeux d'aujourd'hui.

Qualifié, en sous-titre, de «biographie intellectuelle et artistique de Pierre Vadeboncœur", Un moderne à rebours constitue en fait une relecture de l'ensemble de l'œuvre de l'essayiste, incluant quelques textes des années 1940 publiés dans Le Quartier latin et L'Action nationale, en fonction de l'histoire des idées dans laquelle cette œuvre s'est inscrite. Le livre se divise ainsi en trois grandes thématiques - le rapport de Vadeboncœur à l'histoire universelle; celui qu'il entretient avec le passé canadien-français; et l'art et l'enfance dans ses essais - qui structurent autant de chapitres de façon semblable: survol sociohistorique et revue des idées dominantes pertinentes à chacune de ces thématiques au cours des années de formation de l'essayiste, suivi d'une lecture chronologique des essais opportuns. Le procédé, pour être efficace et rigoureux, finit par donner une impression de redite à laquelle un surcroît de concision aurait pu remédier. Cette première réserve n'est pas que cosmétique. Car cette impression de redite, c'est aussi la thèse principale de l'ouvrage et le traitement qu'elle nécessite qui en sont responsables. Voyons plutôt. Si l'on peut parler de «relecture», $c^{\prime}$ est que cette thèse se veut surtout une rectification de la lecture critique dominante. Celle-ci, en effet, a souvent souligné la «ligne de partage» (13), située quelque part dans les années 1970, séparant un premier Vadeboncœur plus engagé à la fois envers sa société et envers la modernité en général, d'un second - la chose est manifeste dans Les deux royaumes (1978) —, plus volontiers marqué par une méditation sur l'infini et «l'humain» à partir de modèles classiques. Or ces derniers essais, avance Livernois, «[sont] loin de constituer une fuite et une œuvre désenchantée ou réactionnaire» (11). Le défi de l'ouvrage consiste alors à nous convaincre que les attaques répétées de «l'inactuel ${ }^{5}$ » Vadeboncœur contre notre "postmodernité» ne renvoient pas à un simple sentiment antimoderne. Il en va donc de la cohérence du parcours de l'essayiste. À cet effet, le second volet de cette thèse vise à soutenir que le recours au passé, de la part de l'essayiste, est antérieur à cette «ligne de partage», voire qu'une «tension» unissant le passé au présent et à l'avenir, jamais véritablement résolue, court tout au long de l'œuvre. Cette thèse a donc avant tout le mérite d'apporter de précieuses nuances au consensus critique qui semble s'être construit au fil des années, celui d'un Vadeboncœur moderne et engagé dans la cité qui tourne brusquement le dos au monde à partir des Deux royaumes, en 1978, déçu par la modernité qu'il appelait de ses vœux. À cette lecture, quelque peu rigide, Jonathan Livernois oppose l'image d'un Vadeboncœur qui, tel un Chateaubriand québécois, serait pris entre deux «régimes d'historicité », notion de l'historien François Hartog qui sert ici de boussole pour s'orienter dans les différentes représentations qu'induisent la modification du rapport des collectivités aux trois modalités du temps - et à leur poids respectif.

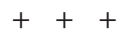

5 Le terme est revendiqué par Vadeboncœur lui-même, dans ses Essais inactuels (Montréal, Boréal, coll. «Papiers collés», 1987, 197 p.). 
En choisissant de prendre pour cible tous les textes importants de l'essayiste s'étant échelonnés sur plus de six décennies d'écriture, Livernois rendait incontournable l'enjeu de la cohérence interne. Confiner la quinzaine d'ouvrages postérieurs aux Deux royaumes à l'étiquette «antimoderne» est la meilleure stratégie pour s'en tenir à La ligne du risque et cantonner Vadeboncœur au chapitre de l'histoire littéraire consacré à la Révolution tranquille. De ce point de vue, la thèse de Livernois est représentative d'une démarche à laquelle la critique doit s'astreindre afin de faire écho à ce qui a mené ce moderne à un tel désenchantement. Si l'ouvrage peine pourtant parfois à emporter notre assentiment, c'est plutôt en raison de sa démonstration, qui, pour être érudite, demeure trop souvent circulaire. Je me limiterai à relever deux exemples.

Le premier argument de Livernois consiste à soutenir que des traces de l'histoire universelle sont présentes dans les essais du premier Vadeboncœur. Si l'argument semble aller de soi, la démonstration, elle, ne va pas sans quelques contorsions. Livernois entreprend ainsi de suivre ces traces laissées par deux périodes historiques, le Moyen Âge et le classicisme, qu'il s'applique d'abord à scruter dans les discours qui circulaient dans l'espace public, et en particulier au Collège Brébeuf, durant les années de formation de l'essayiste. Au moment de La ligne du risque, c'est sans surprise que l'image du Moyen Âge sert à qualifier le passé canadien-français et même son présent, en attente de sa Renaissance pour accéder au «mythe de l'Homme» (90). C'est déjà un changement marquant par rapport à la génération de La Relève qui, à la suite de Jacques Maritain et de Nicolas Berdiaeff, en appelaient à un "nouveau Moyen Âge» (La Relève, cité par Livernois, 82) pour re-spiritualiser la société. Or, c'est pourtant vers un tel sens que l'essayiste se tournera à partir des années 1970. Appelant à « réunir [les] promesses apparemment éteintes » des siècles passés afin de sortir d'un présent jugé catastrophique, Vadeboncœur évoque par exemple, dans Les deux royaumes, le fait que «la Renaissance a étouffé la floraison du Moyen Âge » (cité par Livernois, 96). De telles locutions se multiplient par la suite, au point que Livernois en arrive à les décrire comme « tout droit sorties des années 1930 » (97). Pourtant, l'auteur insiste: « il ne s'agit pas d'une fuite: l'homme est encore de son temps, dans la mesure où il décolle de celui-ci pour mieux l'embrasser et essayer de lui donner, bien modestement, une nouvelle direction» (104). Cet argument, utilisé aussi bien lorsqu'il s'agit de commenter la métaphore dévalorisante du Moyen Âge, dans La ligne du risque (95), que la figure de l'autorité sous l'Ancien Régime vantée par un article du Devoir des années $1930^{6}$, fait de tout un chacun un «inactuel» à partir de la plus simple évocation du passé. En rapprochant certains énoncés de Vadeboncœur de ceux de François Hertel, quand ce n'est pas d'un penseur aussi inquiétant que Berdiaeff, Livernois fait exactement le contraire de ce qu'il prétend: il enfonce l'essayiste dans une rhétorique réactionnaire nostalgique d'une spiritualité perdue.

Le procédé se répète dans le grand chapitre consacré à la fonction de l'art et de l'enfance dans les textes de l'essayiste. D'entrée de jeu, cette fonction est on ne

6 «Un peu comme dans le cas du Moyen Âge, la référence au siècle de Louis XIV permet de donner du jeu à la société canadienne-française, la vivifiant par un air de grandeur et de culture. Desserrant la prise qu'a l'actualité sur leurs vues générales, les deux journalistes adoptent une posture résolument inactuelle. » (110) 
peut plus claire : retourner vers l'enfance serait un moyen de « ralentir la course de la modernité emballée» (248). Ce n'est donc pas un hasard si plusieurs textes de l'essayiste entremêlent fonctions de l'art - en particulier du dessin - et enfance, comme si l'«invisible» que l'œuvre d'art permet de supposer dans sa médiation avec le réel était d'un accès plus facile pour le regard innocent de l'enfant, non encore corrompu par la connaissance du monde - l'influence de Jean-Jacques Rousseau est ici manifeste. Livernois consacre dans ce chapitre de belles pages à la temporalité complexe du dessin, dont le tracé conserve quelque chose du mouvement qui en est l'origine tout en l'inscrivant dans la durée, en particulier celles qui portent sur la «fraîcheur des temps primordiaux» (266) que l'on retrouve dans les œuvres de ces enfantsartistes auxquels l'essayiste s'est plusieurs fois intéressé (Un amour libre, Dix-sept tableaux d'enfant et Qui est le chevalier?). Cet accès à l'éternité est cependant toujours éphémère, condamné par la course du temps dont l'œuvre peut miraculeusement offrir un «concentré» (242), sans pour autant préserver l'artiste de ses effets. Les choses se compliquent toutefois lorsqu'il s'agit de prêter une signification à cette course à rebours par le biais de l'œuvre ou de l'enfance. S'il s'agit de montrer qu'en se réfugiant dans le passé, l'essayiste ne renie pas la modernité mais l'emmène avec lui pour mieux la «structur[er], [la] stabilis[er]» (317), on peut questionner la pertinence de lire la production du collégien des années 1930, pleine des poncifs de son milieu sur les « cimes inaccessibles» (275) offertes par Claude Debussy ou Frédéric Chopin, ou celle des années 1940, afin d'y voir la préfiguration de certains textes écrits à un âge avancé ${ }^{7}$. C'est qu'encore une fois, le commentaire rattache ces développements ultérieurs à une « résurgence du passé de l'essayiste» (287), ce qui le conduit à retrouver derrière les considérations de Vadeboncœur sur le dessin celles d'un essai de Maurice Gagnon publié en 1945, voire de Jacques Maritain (301). L'auteur en conclut que "Vadeboncœur demeure à plusieurs égards un Canadien français tant son discours est ancré dans une pensée qui a ses origines dans les années 1930 et 1940 » (305).

L'ouvrage de Réjean Beaudoin procède d'une façon inverse. Ne cherchant pas à rendre le dernier Vadeboncœur plus fréquentable, il s'évertue plutôt à montrer pourquoi ses derniers textes «antimodernes» sont reçus avec la politesse qui est due à la posture de l'essayiste dans le champ littéraire, à la fois consacré et ignoré, sans qu'ils soient véritablement lus, et pour quelles raisons - essentiellement les mêmes - il est impératif de les lire. Le cœur de l'ouvrage vise donc à prendre au sérieux l'épithète "prophétique» souvent rattachée à l'œuvre, tout en en explicitant le sens. À cet égard, il s'agit davantage d'un essai que d'un commentaire, même s'il prend la forme de ce dernier, aussi bien par l'investissement de l'auteur que par sa visée: renouer un dialogue avec une pensée devenue, jusqu'à un certain point, irrecevable.

7 «Nous l'aurons noté en lisant ces deux textes de 1944 et de 1949 et en les comparant à ceux que l'essayiste publiera 40 ans plus tard: tout se passe comme si rien de fondamental n'avait changé dans la vision de l'art et de son rôle. » (289) 
D'entrée de jeu, Beaudoin situe son essai au carrefour du deuil de l'ami perdu et d'un sentiment imprévu «d'allégresse» (11) né de sa présence irrécusable à la relecture de ses textes, point de départ d'une démarche qu'il qualifie de «dialogue avec [soi-même], mais par l'intermédiaire d'une autre voix» (62). Le principe de lecture est clair : l'adhésion entière à la pensée de l'ami - même si elle sera ici et là mise à rude épreuve.

L'une des forces de l'essai de Beaudoin réside dans la décision d'envisager l'art de Vadeboncœur à partir de ses empêchements ou de ses impasses. Regarder l'œuvre comme un ready-made, comme l'expose l'essayiste dans La clef de voûte, permet de voir «à nu » son passage de «l'ordre des choses périssables » à celui d'une «résistance au temps» $(C V, 155)$. Il suffit pour cela que l'objet soit "posé», ou exposé par une décision. L'art ainsi défini est ouverture vers un autre temps. Cette définition minimale de l'art permet de circonscrire l'impasse initiale à laquelle il est chargé de faire face : le temps fuit; et d'autant plus pour celui qui l'éprouve que l'histoire semble bloquée. Ces deux cas de figure nous mettent sur la piste des deux scènes scrutées par Beaudoin en rapport avec cet enjeu du temps, les scènes ontologique et politique.

Commençons par la première, puisqu'il en va de l'axiome de l'essai de Beaudoin: «ce dont il s'agit, dans les écrits sur l'art, c'est de métaphysique» (59). La question primordiale posée par l'art est celle de cette résistance au temps : comment s'effectue cette résistance, pour qui, et de quelle manière y accéder? Deux portes d'entrée sont ici indiquées par l'auteur: celle de l'émotion ressentie par le spectateur et celle de la nature même de l'œuvre. Dans le premier cas, il s'agit de poser en principe que «le visible offert n'explique pas le sentiment» ressenti (67); il faut pour cela présupposer un invisible, qui tient à la fois de la "genèse de son apparition » et de la conscience de la «conservation du Sens » (68), car l'art «se met devant ce qui est mais va cesser d'être. Il se place en travers de l'ouverture tournée vers le néant» (Vadeboncœur cité par Beaudoin, 69). Ce qui est alors saisi par le spectateur de cette présence qui s'interpose est qualifié d'« existence indirecte» (74), c'est-à-dire appréhension d'une durée qui résiste à la fuite du temps et qui ouvre une voie vers ce qui dépasse sa propre finitude. Il n'en demeure pas moins que cette présence n'est pas donnée, ni pour le spectateur/lecteur ni pour l'artiste, qui doit en passer par des subterfuges qui n'excluent pas l'artifice. L'examen des limites de l'art effectué par Vadeboncœur dans Le pas de l'aventurier, d'autant qu'il procède pour ainsi dire «à rebours de soi» (40), se voit par conséquent longuement interrogé par Beaudoin. La question pourrait être la suivante: si l'œuvre s'interpose face au néant, comment se fait-il que le poète qui s'est approché au plus près de l'absolu, Rimbaud, ait cessé de "s'occuper de ça ( (41)? La réponse est connue, certes, bien qu'elle n'apporte aucun éclairage à l'énigme qu'elle constitue: Rimbaud aurait refusé le bavardage nécessaire au poème, au mensonge que celui-ci doit emprunter pour frayer avec l'inconnu. Ainsi, dans la mesure où l'œuvre de Rimbaud doit être comprise comme une «rébellion poétique», «il n'y a pas vraiment rupture dans le silence final, mais plutôt conjonction profonde avec l'œuvre écrite» (42).

Cette question des limites de l'œuvre est reprise dans le très beau chapitre pénultième. En effet, Qui est le chevalier ? interrogeait déjà l'abandon d'une démarche créatrice à travers la figure d'un artiste précoce qui mit fin à son œuvre dans la 
vingtaine. Mais comme le postule ici Beaudoin, cet essai est surtout l'occasion d'un «métatexte» dans lequel lui-même effectue une sorte d' «exégèse de la pensée de Vadeboncœur» (176). En tant qu' « artiste tombé d'ailleurs dans ce monde-ci», le chevalier rencontre cette fois le mur, « désespérant[,] de la surmodernité» (176). Or, c'est en suivant de près l'argument qui lui est consacré dans L'humanité improvisée que Beaudoin parvient à traiter de cette critique du monde contemporain d'une manière éclairante et propre à la réinscrire dans la discussion sur la culture et le politique que les imprécations de l'essayiste donnaient parfois l'impression d'empêcher ${ }^{8}$. À en croire ce dernier, la postmodernité trouverait son origine dans la révolution qui a refusé de se produire, sauf par une logorrhée devenue usine de production de slogans, lors de Mai-68. Le mouvement qui avait mené une génération au seuil de la rupture s'étant produit au moyen d'une liquidation de son passé, la postmodernité consisterait en ce présentisme aujourd'hui dominant, condamné à sans cesse rejouer cet inadvenu, à «recondui[re] le non-lieu de Mai-68 en forme de statu quo» (85). Même si l'on peut refuser cette identification un peu courte entre une génération précise, celle de Mai68 , et une crise de la mémoire qui d'ailleurs ne l'avait pas attendue pour se produire, en France comme ailleurs ${ }^{9}$, on ne peut qu'être d'accord avec le constat qui accompagne cette critique quant à la confusion actuelle entre liberté et tyrannie propre au fantasme et au caprice.

S'il fallait trouver une constante dans l'œuvre de Vadeboncœur, ce serait sa résistance aux différents blocages de l'avenir qui ont ponctué l'itinéraire de l'auteur, de la société prémoderne du Québec des années 1950 à la «surmodernité» contemporaine. C'est à ce dernier «blocage» que Réjean Beaudoin oppose le «messianisme», défini, dans le sillage de Walter Benjamin et de Giorgio Agamben, en tant que «pratique des temps ouverts par le moyen de l'écriture quand toutes les voies sont barrées» (36). Il ne faut sans doute pas chercher plus loin la raison de la décision de Vadeboncœur de délaisser l'action, syndicale et politique, pour l'écriture, décision progressive qui va de pair avec un diagnostic sur les temps présents et dont le procès devance de beaucoup Les deux royaumes, qui en explicite les motifs. De ce procès difficile témoigne une courte mais exemplaire correspondance de l'écrivain avec Jean-Marc Piotte entre 1963 et $1972{ }^{10}$, correspondance que le hasard du calendrier éditorial a rendue publique en même temps que les deux ouvrages dont il était ici question. Les premiers échanges témoignent de l'effervescence de la Révolution tranquille et jettent un éclairage des plus intéressants sur les débats ayant conduit à la création de la revue Parti pris, dont

8 En particulier lorsqu'il condamne en bloc ce qu'il appelle le «fatras» de cette «surmodernité » : «[E]lle est partout, dans les médias, dans les mœurs, dans les comportements, dans le verbiage, dans des abîmes d'inconscience, et parfois jusque dans la philosophie.» $(C V, 146) \quad 9$ Une part du malaise ressenti à la lecture de cette critique vient du fait que la postmodernité, ou ce qu'elle recouvre, est présentée par Vadeboncœur comme un choix, alors qu'il s'agit plutôt du résultat d'un processus historique commencé il y a longtemps. 10 Jean-Marc Piotte et Pierre Vadeboncœur, Une amitié improbable. Correspondance 1963-1972, présentation de Jacques Pelletier, Montréal, Lux Éditeur, coll. «Lettres libres», 2012, 94 p. 
Piotte était alors «le principal - sinon le seul - véritable "politique"» (15), selon le mot de Jacques Pelletier, débats auxquels les Vadeboncœur, Ferron et Miron participaient de manière occasionnelle. Trait intéressant à relever, si Piotte, qui énonce d'entrée de jeu son admiration pour l'essai éponyme du recueil de 1963, «La ligne du risque», s'attache rapidement à la personnalité de Vadeboncœur, c'est entre autres en raison de sa manière de discuter, lors de ces assemblées officieuses. Lui faisant part d'un malaise provoqué par ses interventions, Piotte y va d'une comparaison fort révélatrice avec Gaston Miron, de la même génération que l'essayiste: "C'est peut-être aussi parce que Miron, lors des rencontres, s'intéresse aux questions qui nous relient, tandis que vous insistez sur ce qui nous sépare.» (28) C'est donc sur ces bases, celles d'un dialogue fondé sur l'écart des perspectives plutôt que sur les consensus, pourtant nombreux, que se construit un échange de plus en plus amical au sujet des aléas de la revue, puis de la situation de la gauche, au Québec, en particulier de leur parti pris pour une action syndicale directement politique, considérée plus efficace que la pureté révolutionnaire d'une gauche radicale alors en plein processus de morcellement.

Dès 1967, l'effervescence est chose du passé. Alors que Piotte rédige à Paris une thèse sur Gramsci, Vadeboncœur lui écrit une longue lettre qui a valeur de diagnostic sur une situation politique d'ores et déjà inquiétante, dans laquelle il énumère une série de traits préfigurant une avancée de la « réaction » : le soutien de nombreux intellectuels à la campagne de Trudeau à la direction du PLC, le clivage qui se fait jour à la CSN, etc. Devant cet essoufflement de la gauche (en partie causé par son éparpillement), Vadeboncœur se tourne de plus en plus ouvertement, dès 1968, vers une forme de "contemplation » qui le conduit à pratiquer ce qu'il appelle une "prose amoureuse» (69) derrière laquelle se laissent deviner certains de ses textes des années 1980. La direction de son travail, deux mois plus tard, semble déjà arrêtée:

\footnotetext{
Pour ma part, étant tant soit peu écrivain, je file lentement ma toile comme une araignée du soir - espoir. Trois ou quatre soirs par semaine, j'y reviens, je trouve lentement images, sens nouveaux; qu'est-ce que c'est? Peu de chose. Mais autant que ce que font les insectes et par la même nécessité qu'eux. Il s'agit de le faire avec une longue, une très longue félicité (70).
}

L'espoir, car il s'agit bien toujours de lui, sera donc soluble dans la longue durée de l'écriture, depuis la solitude de sa toile et en attente, pour ainsi dire, de son lecteur venant s'y prendre, à son corps défendant. 